\title{
Prenatal low-dose bisphenol A enhances behavioral responses induced by a predator odor
}

\author{
Tetsuya Fujimoto', Kazuhiko Kubo ${ }^{2}$, Yasuo Nishikawa ${ }^{1}$ and Shuji Aou ${ }^{3}$ \\ 'Department of Physiology, Osaka Dental University, 8-1, Kuzuha-Hanazono-cho, Hirakata-shi, Osaka, 573-1121, Japan \\ ${ }^{2}$ Department of Otorhinolaryngology, Graduate School of Medical Sciences, Kyushu University, \\ 3-1-1, Maidashi, Higashi-ku, Fukuoka-shi, Fukuoka, 812-8582, Japan \\ ${ }^{3}$ Deptartment of Human Intelligence Systems, Kyushu Institute of Technology, \\ 2-4, Hibikino, Wakamatsu-ku, Kitakyushu-shi, Fukuoka, 808-0196, Japan
}

(Received May 13, 2015; Accepted June 23, 2015)

\begin{abstract}
Bisphenol A (BPA) is an environmental endocrine disrupter (EED). Previous studies by our group showed that pre- and postnatal administration of low-level BPA induced depression-like behavior in rats. In this study, we evaluated the effects of prenatal BPA on behavioral responses to a predator odor by using a novel cross-form apparatus consisting of 4 plastic chambers. On the first day, nothing was placed into the chambers (Session 1). On the second day, a predator odor (fox odor) was located in separate chambers at 2 opposite corners of the apparatus (Session 2). Pregnant Wistar rats were exposed to low-dose BPA (less than the reference dose) during the 7 days just before birth, and the offspring of the treated rats were evaluated as adults. The locomotor activity and avoidance response of each rat on both test days were compared. The control and BPA groups showed reduced locomotor activity in the presence of the predator odor, but the odor-avoidance response was significant only in the BPA rats. The BPA-exposed rats were obviously sensitive to the predator odor. These results suggest that prenatal BPA exposure has an amplifying effect on avoidance responses to predator odor stress.
\end{abstract}

Key words: Bisphenol A, Behavioral toxicity, Prenatal exposure, Predator odor, Avoidance response

\section{INTRODUCTION}

Bisphenol A (BPA) is an environmental endocrine disrupter (EED) that is released from widely used polycarbonate plastics and some dental sealants (Krishnan et al., 1993; Sohoni and Sumpter, 1998; Olea, 2000; Suzuki et al., 2000; Brede et al., 2003; Kang et al., 2003). We have focused on the impact of low-dose BPA on the central nervous system (CNS), especially during the perinatal period. Our group demonstrated that perinatal BPA exposure mitigates gender differences in locomotion, exploratory behavior, and the size of the locus coeruleus (LC) in the brain stem of the rat (Kubo et al., 2001, 2003). We also studied the effects of prenatal (Fujimoto et al., 2006) and postnatal (Fujimoto et al., 2013) BPA exposure on emotion-like behavior by using the forced swimming test (FST) and found a longer average immobility time in BPA-exposed rats. Recent studies from Xu's group in mice have also shown increased immobility time after perinatal exposure to BPA (Xu et al., 2012, 2015).
These results suggest that BPA influences the CNS in a manner that modulates depressive-like behavior.

In addition, fear-related behaviors were altered by BPA in mice (Matsuda et al., 2013) and rats (Negishi et al., 2004). In the elevated plus maze (EPM), exposure of rats to BPA produced anxiogenic effects (Patisaul and Bateman, 2008; Patisaul et al., 2012; Diaz Weinstein et al., 2013; Zhou et al., 2015), anxiolytic effects (Farabollini et al., 1999; Fujimoto et al., 2013; Kuwahara et al., 2014), or unclear/nonexistent effects (Negishi et al., 2004; Fujimoto et al., 2006; Jones and Watson, 2012; Kuwahara et al., 2013). The different reported effects of BPA in rats in the EPM might be due to differences in experimental design or environmental conditions.

There is no doubt that BPA interacts with the neural basis of emotion-related behaviors, but the mechanisms underlying this interaction are not well understood. In this study, we investigated the behavioral response to a predator odor (2,4,5-trimethylthiazoline, TMT) by using a new cross-form apparatus that partially resem-

Correspondence: Tetsuya Fujimoto (E-mail: tetsu-f@cc.osaka-dent.ac.jp) 
bled the EPM. Predator odors, such as those from cats or foxes, elicit several emotion-like responses in rodents (Dielenberg and McGregor, 2001; Blanchard et al., 2003). TMT is a substance found in fox feces that has been used extensively in animal research since the first report of behavioral and hormonal responses to TMT in rats (Vernet-Maury et al., 1984). Generally, TMT increased freezing and avoidance responses (Vernet-Maury et al., 1984; Morrow et al., 2000; Fendt et al., 2005; Fendt and Endres, 2008), as well as corticosterone release (Vernet-Maury et al., 1984; Morrow et al., 2000). Recent studies reported that low-dose BPA altered the hypothalamic-pituitary-adrenocortical (HPA) axis and found high plasma corticosterone levels and low glucocorticoid receptor levels in the brains of BPA-treated rats (Poimenova et al., 2010; Panagiotidou et al., 2014; Zhou et al., 2015). We focused on the manner in which BPA modified behavioral responses elicited by TMT, because modification of such fight-or-flight responses, whether via amplification or attenuation, implies alteration of neural systems such as the HPA axis.

\section{MATERIAL AND METHODS}

\section{Animals}

Female and male adult rats (Kud:Wistar, Kyudo Corp, Saga, Japan) were housed in our laboratory for 1 week. After copulation, 5 mother rats were exposed to vehicle as a control treatment, and another 5 dams were exposed to BPA. The animals were kept in a closed colony room under a 12:12-hr light-dark cycle (light period, 08:0020:00 hr) in a constantly air-conditioned environment $\left(23 \pm 1{ }^{\circ} \mathrm{C}, 60 \pm 10 \%\right.$ relative humidity). The animals had free access to laboratory chow (CE-2, CLEA Japan, Inc., Tokyo, Japan) and tap water. All experiments were performed under the Guidelines for Animal Experiments of the Graduate School of Life Science and Systems Engineering, Kyushu Institute of Technology and pertinent governmental regulations (Law No. 105 and Notification No. 6) of Japan.

\section{Treatment}

BPA was purchased from Sigma-Aldrich Co. (St. Louis, MO, USA) and administered to the rats via drinking water in glass bottles. All dams were given tap water until gestational day 13, at which time the BPA group was administered BPA solution (0.1 ppm in distilled water) and the control group was administered vehicle (distilled water). Exposure to BPA or vehicle was continued until the day of birth (postnatal day 0, PND 0). The average BPA intake (calculated from body weight and water intake) was $15 \mu \mathrm{g} / \mathrm{kg} /$ day. On PND 1, the litters were balanced by removing pups, so that each contained 4 males and 4 females. The pups in each group were selected by body weight. First, runts, if any, were removed, and the average weight of sexes was determined. Four male and 4 female offspring were selected for each group based on the similarity of their weights to the average weight. On PND 21, all offspring were weaned, classified, and housed in groups consisting of rats of the same sex and from the same litter, and the rats were allowed access ad libitum to food and tap water until the end of the experiment.

\section{Behavioral tests \\ General}

Adult offspring of the BPA- and vehicle-treated dams (10-14 weeks of age) were used for this study (1-4 animals from each dam and sex group were selected). There were 13 male control rats, 14 female control rats, 13 male BPA-treated rats, and 14 female BPA-treated rats. In this study, gender differences were not considered, so data from male and female rats in each treatment group were considered together (27 control rats and 27 BPA-treated rats). All sessions were automatically recorded with a computer-based videotracking system (Ethovision version 1.96, Noldus Info. Tech., Wageningen, Netherlands).

\section{Cross-form apparatus}

The apparatus consisted of 4 plastic chambers $(45 \times 15$ $\times 15 \mathrm{~cm})$. The open side of each chamber was arranged to face the same area, so that the chambers formed a cross (Fig. 1a). On the CCD-camera view, the left, upper, right, and bottom chambers were designated as Chamber A, Chamber B, Chamber C, and Chamber D, respectively. The locations of the chambers were kept consistent between experiments by using marks placed at specific locations on the laboratory floor as guidelines for chamber placement.

\section{Procedures}

The experiment was conducted in 2 sessions (Fig. 1). On the first day, all chambers were empty, and the behavioral parameters of each animal were recorded after it was placed into the apparatus (Session 1, Fig. 1a). On the next day, the odor solution was placed into Chamber A and Chamber C, while the odor solvent only was placed into Chamber B and Chamber D. The behavior of each animal in the apparatus was recorded again, and behavior in each session was compared (Session 2, Fig. 1b). All chambers were washed before every test session. In addition, to further to exclude the potential influence of remaining odors, the chambers used for Session 2 were never used for Ses- 

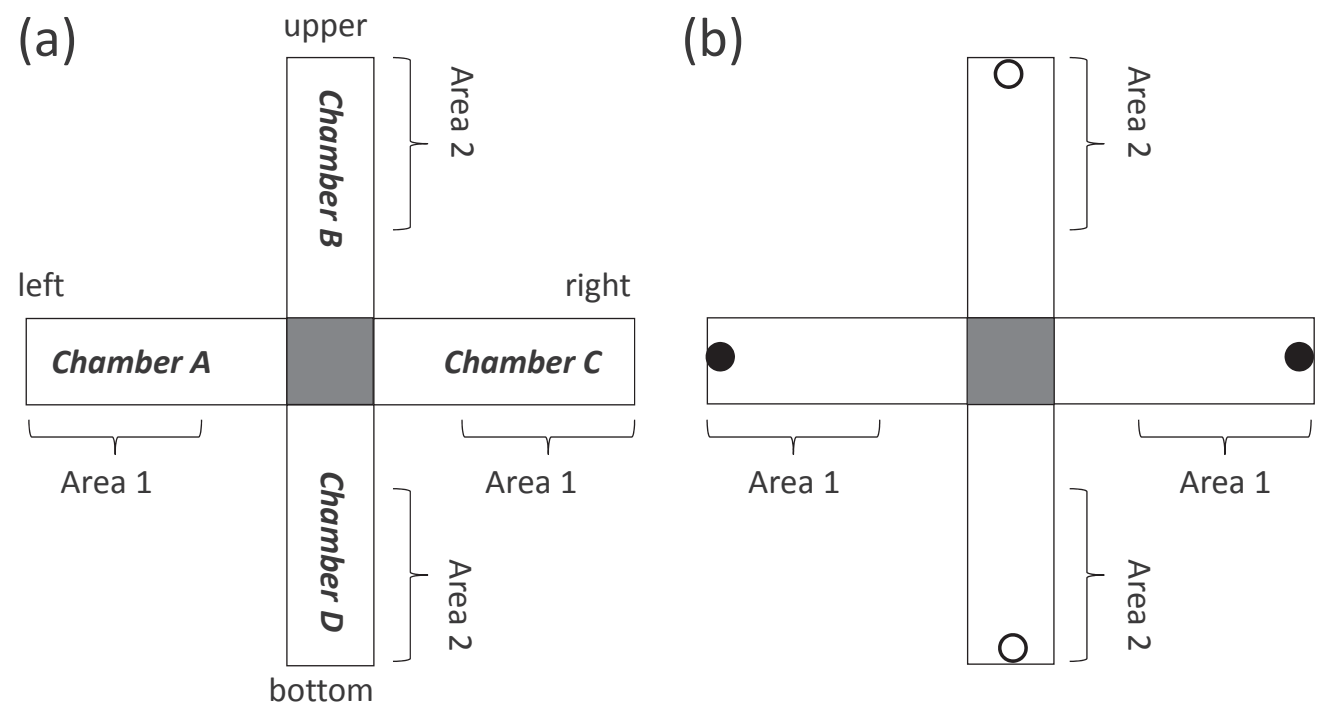

Fig. 1. The cross-form apparatus consisted of 4 plastic chambers $(45 \times 15 \times 15 \mathrm{~cm})$. The open sides were arranged to face each other to form a cross. On the CCD-camera view, the left, upper, right, and bottom chambers were designated as Chamber A, Chamber B, Chamber C, and Chamber D, respectively. The central gray area represents the open space between the chambers. The experiment was conducted in 2 sessions. (a) Session 1: on the first day, all chambers were empty, and the animals were placed into the central open space for recording of behavioral parameters. (b) Session 2: the next day, the odor solution (black circles) was placed at the back of Chamber A and Chamber C, while the solvent only (white circles) was placed at the back of Chamber B and Chamber D. The animals were placed into the chambers again and their behaviors were recorded. TMT (fox odor) was used as the odor solution. Triethyl citrate, an odor-free oil, was used as the solvent. Three areas were considered by the software (Ethovision version 1.96) for this experiment: whole area, the area of the entire apparatus; Area 1, the area extending $30 \mathrm{~cm}$ from the back of Chambers A and C; and Area 2, the area extending $30 \mathrm{~cm}$ from the back of Chambers B and D.

sion 1. In Session 2, the chambers used for the odor solution were never used for the odor solvent, and vice versa.

\section{Odor solution}

TMT (fox odor) was supplied by Contech Inc. (Victoria, BC, Canada). Triethyl citrate, odor-free oil, was supplied by the Tokyo Chemical Industry Co., Ltd. (Tokyo, Japan) and used as the odor solvent. Solutions of 3\% TMT (odor solution) and triethyl citrate (solvent only) were prepared, and $0.05 \mathrm{~mL}$ of each solution was dropped onto a piece of cotton in a $15 \mathrm{~mL}$ glass vial for addition to the appropriate chamber during Session 2.

\section{Behavioral parameters}

Each rat was placed on the center space of the crossform apparatus and was allowed to move freely for $10 \mathrm{~min}$. We recorded 1) total distance moved, 2) rapid movement (time during which the animals moved at a rate of $10 \mathrm{~cm} / \mathrm{sec}$ or more), and 3) time spent in each area of the apparatus. These movement parameters were recorded in the following areas: 1) the whole area, the entire area of the apparatus; 2) Area 1, the areas extending $30 \mathrm{~cm}$ from the backs of Chambers A and C; and 3) Area 2, the areas extending $30 \mathrm{~cm}$ from the backs of Chambers B and D (Fig. 1). For the time spent in each area, the ratio of time spent in Area 1 to the total time (\%) was calculated by the following formula: (Area 1)/(Area $1+$ Area 2$) \times 100$. Total distance moved and rapid movement were evaluated as indices of locomotor activity. The time spent in each area was evaluated as an avoidance response to the predator odor. The same animals were tested during Session 1 and Session 2, and the results from Session 1 (without odor exposure) were used as control data for Session 2.

\section{Statistical analysis}

Paired $t$-tests were performed for comparisons of Session 1 behavior vs. Session 2 behavior within each animal group. Student's $t$-tests were performed to assess differences between 2 animal groups (control vs. BPA) within the same session. All data were analyzed using Statview 
(a)

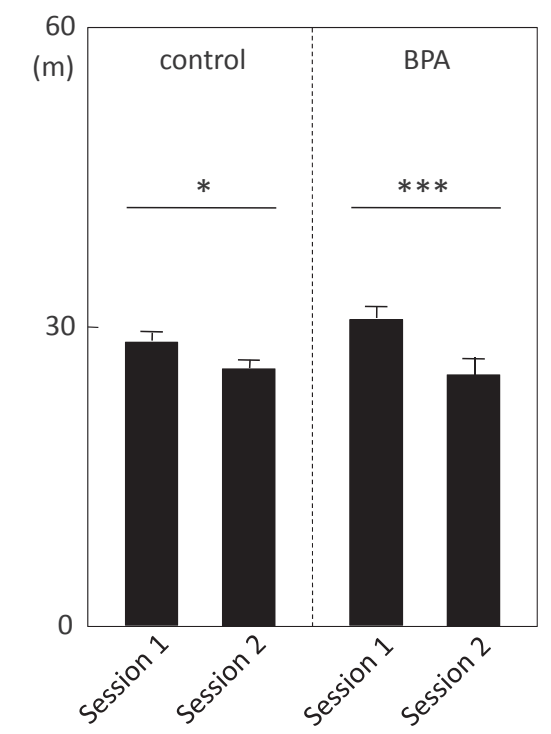

(b)

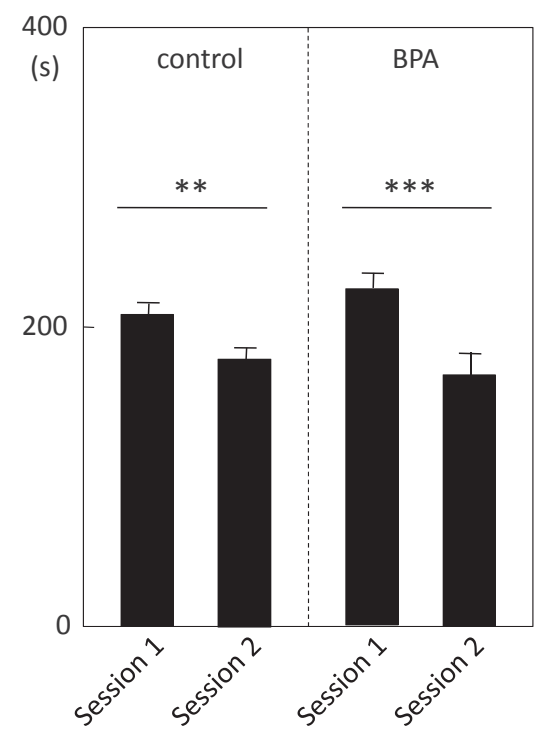

Fig. 2. Locomotor activity results in the whole apparatus area. (a) Total distance moved (in meters) and (b) time spent in rapid movement (in sec) are presented. The columns represent mean \pm S.E.M. ( $\mathrm{n}=27$ animals per group). $* P<0.05, * * \mathrm{P}<0.01$, $* * * P<0.001$ (paired $t$-test vs. Session 1).

5.0 software for Macintosh (SAS Institute Inc., Cary, NC, USA). Statistical differences were considered significant when the $P$-value was less than 0.05 . All results are presented as mean \pm standard error of the mean (S.E.M.).

\section{RESULTS}

The total distance moved and rapid movement in the whole apparatus area are presented in Fig. $2 \mathrm{a}$ and $2 \mathrm{~b}$, respectively, as measurements of locomotor activity. The control and BPA groups displayed significantly decreased scores in Session 2 (under exposure to TMT odor) (vs. Session 1, paired $t$-test). In both sessions, significant differences were not identified among the BPA and control groups. As measurements of the avoidance response to the predator odor, the time spent in Areas 1 and 2 is presented in Fig. 3a and 3b, whereas the ratio of the time spent in Area 1 to the time spent in (Area $1+$ Area 2) is shown in Fig. 3c. In time spent by the BPA-treated rats in Area 2 was increased in Session 2 in comparison with Session 1 (Fig. 3b). The staying rate (ratio, \%) of the BPAtreated rats in the odor area was decreased in Session 2 in comparison with Session 1 (Fig. 3c). The behavior of the control group did not differ significantly among the test sessions (paired $t$-test). The BPA-treated group spent sig- nificantly more time than the control group in Area 2 during Session 2 (Fig. 3b).

\section{DISCUSSION}

Although, behavioral responses to TMT odor in rodents have been well investigated (Vernet-Maury et al., 1984; Morrow et al., 2000; Fendt et al., 2005; Fendt and Endres, 2008), to our knowledge, this is the first study in which TMT stimulus has been used for evaluating the perinatal exposure of BPA. As described before, BPA has been studied using the EPM method, which is typically used for evaluating anxiety; but those results were various by the study groups, and our results were also unclear (Fujimoto et al., 2006, 2013). In this study, we used TMT odor to stimulate anxiety, and used new apparatus similar to EPM. We found enhanced responses to predator odor in the rats that were prenatally exposed to BPA.

The total distance moved (Fig. 2a) and rapid movement (Fig. 2b) were generally decreased by exposure to TMT. Locomotor behaviors in response to the predator odor were similar in the control and BPA groups: both control and BPA rats showed decreased locomotor activity in the presence of TMT. The effects of TMT on avoidance responses (Fig. 3) were generally mild in comparison 
Bisphenol A enhances the response to a predator odor

(a)

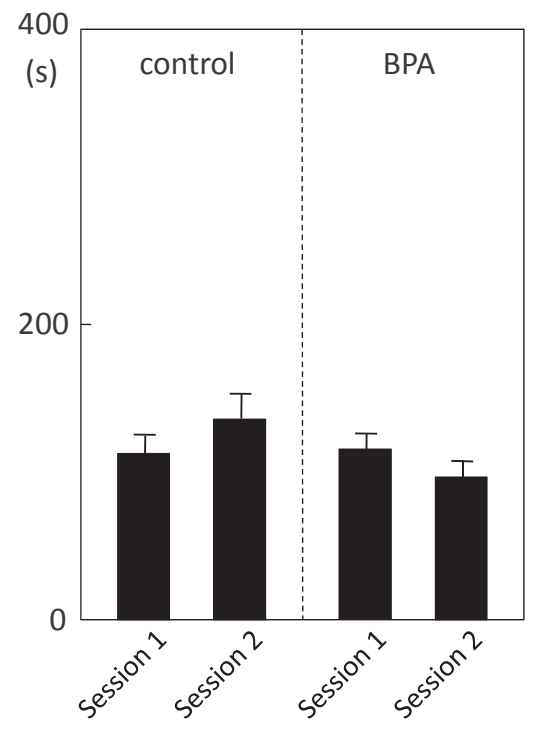

(b)

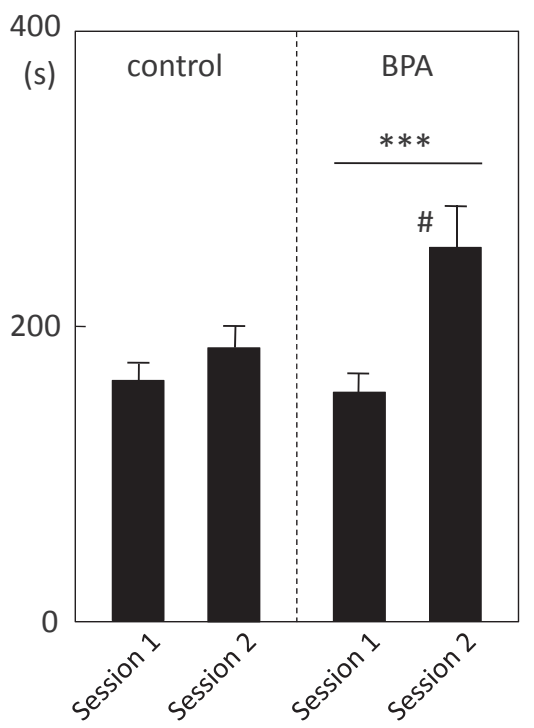

(c)

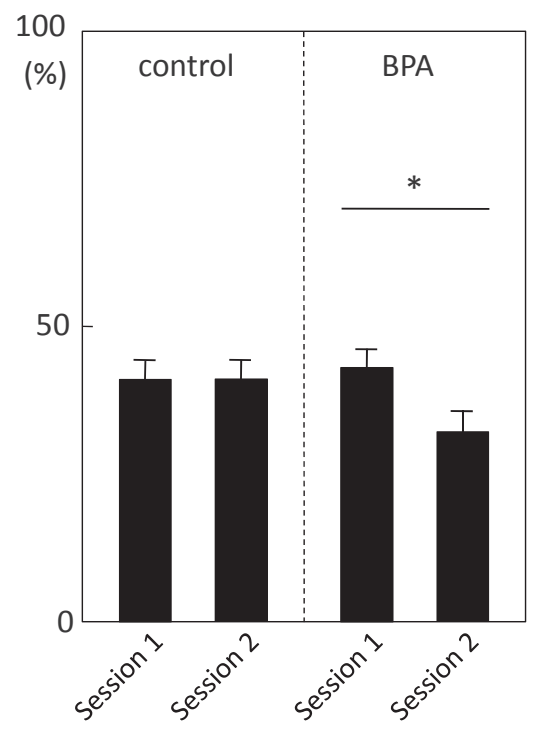

Fig. 3. Time spent in (a) Area 1 and (b) Area 2 (in sec), and (c) the ratio (\%) of time spent in Area 1 to the total time ((Area 1)/ $($ Area $1+$ Area 2$) \times 100)$. The columns represent mean \pm S.E.M. $(\mathrm{n}=27$ animals per group). $* P<0.05, * * * P<0.001$ (paired $t$-test vs. Session 1). \# $P<0.05$ (Student's $t$-test vs. control animals during the same session).

with its effects on locomotor activity. The control group did not show any significant effects of TMT. In the measurements of time spent in each area, significant effects of TMT were found only in BPA rats. The BPA rats showed increased time spent in Area 2 in Session $2(P<0.001$ vs. Session $1, P<0.05$ vs. control, Fig. 3b), suggesting that these rats avoided Area 1 (with the TMT odor) and moved into Area 2 (with no odor). This result was reflected in the ratio of the time spent in each area (\%) (Fig. 3c). As shown in Figs. 2 and 3, the predator odor used in the present study decreased locomotor activity in control rats, but did not change avoidance behavior. In contrast, BPA rats showed a significant response to predator odor in both locomotor and avoidance behaviors (Fig. 2, Fig. 3). A previous review indicated that TMT produced fear behavior (freezing) at lower concentrations than were required to produce avoidance behavior (Fendt and Endres, 2008). In our study, we did not evaluate freezing behavior, but we showed that locomotor activity was also sensitively affected by TMT exposure. Our results indicate that BPA clearly increased TMT avoidance behavior and had a small impact on the animals' susceptibility to TMT-associated disturbances in locomotor activity.

Although we did not measure stress hormone levels in this study, it has been established that predator odors increase corticosterone levels (Adamec et al., 1998; Vernet-Maury et al., 1984; Morrow et al., 2000). In addi- tion, EPM studies show that predator odors increase anxiety levels in rats (Adamec and Shallow, 1993) and mice (Calvo-Torrent et al., 1999). Dielenberg et al. (1999) reported that avoidance of cat odor by rats was attenuated by anxiolytic drugs. These reports demonstrate that predator odors are stressful for animals, as indicated by elevated levels of anxiety-like behavior and clear avoidance of predator odors. Recent studies showed that low-dose BPA altered the HPA axis, leading to increased plasma corticosterone levels and decreased glucocorticoid receptor levels in the brains of BPA-treated rats (Poimenova et al., 2010; Panagiotidou et al., 2014; Zhou et al., 2015). These reports suggest that BPA induced neural alterations associated with the HPA axis, leading BPA rats to feel anxiety and avoid TMT.

In a previous study, we showed that prenatal BPA treatment enhanced the depression-like response in the FST (Fujimoto et al., 2006). Because the causes of mental illness such as depression are not well understood, recent studies have focused on the interaction between stressful life-events and the serotonin system. Individuals with low serotonin transporter (SERT) function showed susceptibility to stressful life-events and were more likely to develop depression (Caspi et al., 2003; Kendler et al., 2005). In a study using SERT knockout mice and the EPM, anxiety-like behavior was enhanced under exposure to cat odor only in the knockout mice (Adamec et al., 2006). 
These reports clearly demonstrate that predator odors could alter emotion-like behaviors. Taken together with previous reports, our findings suggest that BPA increased the susceptibility of rats to TMT odor and possibly to other stressful stimuli. We suspect that the observed change in emotional status was similar to that underlying the prolongation of immobility in the FST observed by our group (Fujimoto et al., 2006), and we suggest that both behavioral findings were probably due to neural changes in the HPA system and/or the serotonin system induced by prenatal BPA treatment. The present study showed the suitability of our evaluation strategy using TMT to detect behavioral changes regarding the weakness to stresses. The testing strategy described herein could be used for psychopharmacological studies using diverse drugs and animal models.

Conflict of interest---- The authors declare that there is no conflict of interest.

\section{REFERENCES}

Adamec, R., Kent, P., Anisman, H., Shallow, T. and Merali, Z. (1998): Neural plasticity, neuropeptides and anxiety in animals--implications for understanding and treating affective disorder following traumatic stress in humans. Neurosci. Biobehav. Rev., 23, 301-318.

Adamec, R., Burton, P., Blundell, J., Murphy, D.L. and Holmes, A. (2006): Vulnerability to mild predator stress in serotonin transporter knockout mice. Behav. Brain Res., 170, 126-140.

Adamec, R.E. and Shallow, T. (1993): Lasting effects on rodent anxiety of a single exposure to a cat. Physiol. Behav., 54, 101-109.

Blanchard, D.C., Griebel, G. and Blanchard, R.J. (2003): Conditioning and residual emotionality effects of predator stimuli: some reflections on stress and emotion. Prog. Neuropsychopharmacol. Biol. Psychiatry, 27, 1177-1185.

Brede, C., Fjeldal, P., Skjevrak, I. and Herikstad, H. (2003): Increased migration levels of bisphenol A from polycarbonate baby bottles after dishwashing, boiling and brushing. Food Addit. Contam., 20, 684-689.

Calvo-Torrent, A., Brain, P.F. and Martinez, M. (1999): Effect of predatory stress on sucrose intake and behavior on the plus-maze in male mice. Physiol. Behav., 67, 189-196.

Caspi, A., Sugden, K., Moffitt, T.E., Taylor, A., Craig, I.W., Harrington, H., McClay, J., Mill, J., Martin, J., Braithwaite, A. and Poulton, R. (2003): Influence of life stress on depression: moderation by a polymorphism in the 5-HTT gene. Science, 301, 386-389.

Diaz Weinstein, S., Villafane, J.J., Juliano, N. and Bowman, R.E. (2013): Adolescent exposure to Bisphenol-A increases anxiety and sucrose preference but impairs spatial memory in rats independent of sex. Brain Res., 1529, 56-65.

Dielenberg, R.A., Arnold, J.C. and McGregor, I.S. (1999): Lowdose midazolam attenuates predatory odor avoidance in rats. Pharmacol. Biochem. Behav., 62, 197-201.

Dielenberg, R.A. and McGregor, I.S. (2001): Defensive behavior in rats towards predatory odors: a review. Neurosci. Biobehav.
Rev., 25, 597-609.

Farabollini, F., Porrini, S. and Dessì-Fulgherit, F. (1999): Perinatal exposure to the estrogenic pollutant bisphenol A affects behavior in male and female rats. Pharmacol. Biochem. Behav., 64, 687-694.

Fendt, M. and Endres, T. (2008): 2,3,5-Trimethyl-3-thiazoline (TMT), a component of fox odor - just repugnant or really fearinducing? Neurosci. Biobehav. Rev., 32, 1259-1266.

Fendt, M., Endres, T., Lowry, C.A., Apfelbach, R. and McGregor, I.S. (2005): TMT-induced autonomic and behavioral changes and the neural basis of its processing. Neurosci. Biobehav. Rev., 29, 1145-1156.

Fujimoto, T., Kubo, K. and Aou, S. (2006): Prenatal exposure to bisphenol A impairs sexual differentiation of exploratory behavior and increases depression-like behavior in rats. Brain Res., $1068,49-55$.

Fujimoto, T., Kubo, K., Nishikawa, Y. and Aou, S. (2013): Postnatal exposure to low-dose bisphenol A influences various emotional conditions. J. Toxicol. Sci., 38, 539-546.

Jones, B.A. and Watson, N.V. (2012): Perinatal BPA exposure demasculinizes males in measures of affect but has no effect on water maze learning in adulthood. Horm. Behav., 61, 605-610.

Kang, J.H., Kito, K. and Kondo, F. (2003): Factors influencing the migration of bisphenol A from cans. J. Food Prot., 66, 14441447.

Kendler, K.S., Kuhn, J.W., Vittum, J., Prescott, C.A. and Riley, B. (2005): The interaction of stressful life events and a serotonin transporter polymorphism in the prediction of episodes of major depression: a replication. Arch. Gen. Psychiatry, 62, 529-535.

Krishnan, A.V., Stathis, P., Permuth, S.F., Tokes, L. and Feldman, D. (1993): Bisphenol-A: an estrogenic substance is released from polycarbonate flasks during autoclaving. Endocrinology, 132, 2279-2286.

Kubo, K., Arai, O., Ogata, R., Omura, M., Hori, T. and Aou, S. (2001): Exposure to bisphenol A during the fetal and suckling periods disrupts sexual differentiation of the locus coeruleus and of behavior in the rat. Neurosci. Lett., 304, 73-76.

Kubo, K., Arai, O., Omura, M., Watanabe, R., Ogata, R. and Aou, S. (2003): Low dose effects of bisphenol A on sexual differentiation of the brain and behavior in rats. Neurosci. Res., 45, 345356.

Kuwahara, R., Kawaguchi, S., Kohara, Y., Cui, H. and Yamashita, K. (2013): Perinatal exposure to low-dose bisphenol A impairs spatial learning and memory in male rats. J. Pharmacol. Sci., 123, 132-139.

Kuwahara, R., Kawaguchi, S., Kohara, Y., Jojima, T. and Yamashita, K. (2014): Bisphenol A does not affect memory performance in adult male rats. Cell. Mol. Neurobiol., 34, 333-342.

Matsuda, S., Matsuzawa, D., Ishii, D., Tomizawa, H., Sajiki, J. and Shimizu, E. (2013): Perinatal exposure to bisphenol A enhances contextual fear memory and affects the serotoninergic system in juvenile female mice. Horm. Behav., 63, 709-716.

Morrow, B.A., Redmond, A.J., Roth, R.H. and Elsworth, J.D. (2000): The predator odor, TMT, displays a unique, stress-like pattern of dopaminergic and endocrinological activation in the rat. Brain. Res., 864, 146-151.

Negishi, T., Kawasaki, K., Suzaki, S., Maeda, H., Ishii, Y., Kyuwa, S., Kuroda, Y. and Yoshikawa, Y. (2004): Behavioral alterations in response to fear-provoking stimuli and tranylcypromine induced by perinatal exposure to bisphenol A and nonylphenol in male rats. Environ. Health Perspect., 112, 1159-1164.

Olea, N. (2000): Bisphenol A and dental sealants: Olea's response. 
Bisphenol A enhances the response to a predator odor

Environ. Health Perspect., 108, A546.

Panagiotidou, E., Zerva, S., Mitsiou, D.J., Alexis, M.N. and Kitraki, E. (2014): Perinatal exposure to low-dose bisphenol A affects the neuroendocrine stress response in rats. J. Endocrinol., 220, 207-218.

Patisaul, H.B. and Bateman, H.L. (2008): Neonatal exposure to endocrine active compounds or an ER $\beta$ agonist increases adult anxiety and aggression in gonadally intact male rats. Horm. Behav., 53, 580-588.

Patisaul, H.B., Sullivan, A.W., Radford, M.E., Walker, D.M., Adewale, H.B., Winnik, B., Coughlin, J.L., Buckley, B. and Gore, A.C. (2012): Anxiogenic effects of developmental bisphenol A exposure are associated with gene expression changes in the juvenile rat amygdala and mitigated by soy. PLoS One, 7, e43890. doi: 10.1371/journal.pone.0043890.

Poimenova, A., Markaki, E., Rahiotis, C. and Kitraki, E. (2010): Corticosterone-regulated actions in the rat brain are affected by perinatal exposure to low dose of bisphenol A. Neuroscience, 167, 741-749.

Sohoni, P. and Sumpter, J.P. (1998): Several environmental oestro- gens are also anti-androgens. J. Endocrinol., 158, 327-339.

Suzuki, K., Ishikawa, K., Sugiyama, K., Furuta, H. and Nishimura, F. (2000): Content and release of bisphenol A from polycarbonate dental products. Dent. Mater. J., 19, 389-395.

Vernet-Maury, E., Polak, E.H. and Demael, A. (1984): Structure-activity relationship of stress-inducing odorants in the rat. J. Chem. Ecol., 10, 1007-1018.

Xu, X., Dong, F., Yang, Y., Wang, Y., Wang, R. and Shen, X. (2015): Sex-specific effects of long-term exposure to bisphenol-A on anxiety- and depression-like behaviors in adult mice. Chemosphere, 120, 258-266.

Xu, X., Hong, X., Xie, L., Li, T., Yang, Y., Zhang, Q., Zhang, G. and Liu, X. (2012): Gestational and lactational exposure to bisphenol-A affects anxiety- and depression-like behaviors in mice. Horm. Behav., 62, 480-490.

Zhou, R., Chen, F., Feng, X., Zhou, L., Li, Y. and Chen, L. (2015): Perinatal exposure to low-dose of bisphenol A causes anxietylike alteration in adrenal axis regulation and behaviors of rat offspring: a potential role for metabotropic glutamate $2 / 3$ receptors. J. Psychiatr. Res., 64, 121-129. 